\title{
Health-Related Quality of Life, Depression, and Smoking Status in Patients with COPD: Results from the Behavioral Risk Factor Surveillance System Data
}

\author{
Jessica Floyd*, Jennifer Mallow, Laurie Theeke \\ School of Nursing, West Virginia University, Morgantown, WV, USA \\ Email: `jmf0002@mix.wvu.edu, jamallow@hsc.wvu.edu, ltheeke@hsc.wvu.edu
}

How to cite this paper: Floyd, J., Mallow, J. and Theeke, L. (2018) Health-Related Quality of Life, Depression, and Smoking Status in Patients with COPD: Results from the Behavioral Risk Factor Surveillance System Data. Open Journal of Nursing, 8, 779-794.

https://doi.org/10.4236/ojn.2018.811059

Received: September 29, 2018

Accepted: November 16, 2018

Published: November 19, 2018

Copyright $\odot 2018$ by authors and Scientific Research Publishing Inc. This work is licensed under the Creative Commons Attribution International License (CC BY 4.0).

http://creativecommons.org/licenses/by/4.0/

\begin{abstract}
Background: Chronic Obstructive Pulmonary Disease (COPD) is a leading cause of mortality. People living with COPD often have a common triad of problems including decreased health-related quality of life (HRQL), smoking, and depression. Identifying barriers to preventing and treating COPD is of the utmost importance. The purpose of this study is to examine the relationship between HRQL, depression, and smoking status for patients with COPD. Methods: The 2016 BRFSS data was used to perform a cross sectional analysis of adult patients with a diagnosis of COPD. A comprehensive descriptive analysis of all study variables for those participants having COPD was performed. Then relationships between general HRQL, depression, and smoking status were examined. Data were analyzed using SPSS. Results: The original 2016 BRFSS dataset contained responses from 486,303 participants. After selecting participants who self-identified as having a diagnosis of COPD, 40,682 individual participants remained in the dataset for further analysis. The participants with COPD were mostly female, over the age of 65 , with low-incomes, attended a year of college or less, with some type of healthcare coverage. Patients with a dual diagnosis of COPD and depression have poorer HRQL and an increased number of cigarettes smoked compared to those patients with COPD. Likewise, there is a significant relationship between HRQL and smoking status for patients with a dual diagnosis of COPD and depression. Conclusion: Depression as a comorbidity does have a statistically significant relationship with patients HRQL and smoking status. Future research should be aimed at increasing screening and treatment for depression in patients with COPD who continue to smoke. Further research on the cyclical rela-
\end{abstract}


tionship between COPD, depression, and smoking cessation would be beneficial.

\section{Keywords}

Pulmonary Disease, Chronic Obstructive, Smoking, Depression, Health-Related Quality of Life

\section{Introduction}

The prevalence of chronic obstructive pulmonary disease (COPD) in the United States is $6.4 \%$, affecting 15.7 million Americans [1]. Prevention and treatment efforts for COPD are well known in the clinical community and are centered on minimizing risk factors, managing symptoms, and reducing exacerbations [1]. However, as a preventable and treatable illness, COPD is the third leading cause of death in the world [2].

The leading modifiable risk factor for developing and/or exacerbating COPD is tobacco smoking behavior [3]. Though it is reported that smoking cessation slows the progression of COPD and decreases COPD symptoms and exacerbations, one third of the COPD population continue to smoke [4]. Continued smoking behavior leads to subsequent exacerbations of COPD which results in additional breathing difficulties that are related to poor physical, mental, and emotional health outcomes.

In the COPD patient population, depression has a prevalence rate of $40 \%$ [5]. Concurrent depression is problematic because it is linked to lack of treatment adherence and magnification of COPD symptoms and exacerbations [6]. Depression as a comorbidity is also troublesome because people living with both COPD and depression are reported to have greater difficulty with smoking cessation [6]. People living with COPD often have a common triad of problems including decreased health related quality of life, smoking, and depression [7].

The purpose of this study is to examine the relationship between health-related quality of life, depression, and smoking status for patients with COPD. The following research questions and hypotheses guided the analysis:

What is the difference in HRQL for COPD patients who have depression when compared to those who do not have depression?

Hypothesis 1: Participants with comorbid depression will report poorer HRQOL.

What is the difference in smoking status in COPD patients who have depression when compared to those who do not have depression?

Hypothesis 2: Participants with depression will report smoking a higher number of cigarettes when compared to those without depression.

What is the relationship between HRQL and smoking status for a subgroup of patients with COPD and Depression?

Hypothesis 3: In this subgroup, patients with smoking status will have poorer 
HRQL.

\section{Methods}

\subsection{Data Source, Study Design, and Ethical Conduct of Research}

Behavioral Risk Factor Surveillance System (BRFSS) data was originally designed to help states collect data so that it was possible to track state and local data for health program planning. Since 1984, the Centers for Disease Control and Prevention (CDC) has conducted a cross-sectional, annual health-related telephone survey in the United States and surrounding United States territories to collect data on health behaviors, chronic diseases, health care accessibility, and the use of health services. The telephone surveys have three components: standardized questions, optional questions, and an opportunity for states to add questions [8]. Currently almost two thirds of US states use this data to provide evidence for health legislation.

A cross-sectional descriptive design was used to answer the research questions. For 2016, the BRFSS questionnaire was developed and subsequently, health departments within the participating locations administered the survey using Computer Assisted Telephone Interview (CATI) systems. In 2016, all 50 states within the United States participated in the BRFSS, as well as, the District of Columbia, Guam, Puerto Rico, and the US Virgin Islands. For landline surveys, a disproportionate stratified sample design was used by the United States and the District of Columbia and a simple random-sample design was used by Guam, Puerto Rico, and the US Virgin Islands. Cellular surveys used the Telecordia database of telephone exchanges and 1000 banks to select telephone numbers at random. Once collected, 2016 data was processed, aggregated, weighted by the CDC, and made publicly available for analysis on the CDC website [8]. The study team retrieved the 2016 BRFSS data and performed an analysis of the relationships between health-related quality of life (HRQL) and smoking status for patients with a dual diagnosis of COPD and depression.

This study is a secondary data analysis of publicly available de-identified BRFSS data. Hence, the research is considered as non-human subjects research (NHSR). As a NHSR study, Institutional Review Board (IRB) approval was not required or sought.

\subsection{Study Population and Sample}

The original 2016 BRFSS dataset included 486,303 participants. For this study, the population of interest was adults who were 18 years and older, not institutionalized and self-identified as having a diagnosis of COPD. After selecting participants based on the listed criteria, 40,682 individual participants remained in the study sample for inclusion in the analysis.

\subsection{Measures}

Descriptive Variables: 


\section{Income}

Participants were asked, "Is your annual household income from all sources": The categorical responses that a participant could choose were: less than $\$ 10,000$; less than $\$ 15,000$; less than $\$ 20,000$; less than $\$ 25,000$; less than $\$ 35,000$; less than $\$ 50,000$; less than $\$ 75,000 ; \$ 75,000$ or more.

\section{Education Level}

Participants were asked to select their "level of education completed". The categorical responses that a participant could choose were: did not graduate high school, graduated high school, attended college or technical school, graduated for college or technical school, don't know/not sure, or missing. Participants were also asked what the highest grade or year of school they completed. They responded by answer: never attended school or only kindergarten, grades $1-8$, grades 9 - 11, grade 12 or GED, college 1 year to 3 years, college 4 years or more, refused, not asked or missing.

\section{Age}

Participants were asked to self-report their age in years. However, the actual age of the participant is not reported in the dataset. The following six weighted age categories were provided in the dataset and were used in this analysis: age 18 - 24, age 25 - 34, age 35 - 44, age 45 - 54, age 55 - 64, and age 65 or older.

\section{Gender}

Participants were asked to categorize their sex as either male or female.

\section{Race}

To determine race, participants were asked, "Which one of these groups would you say best represents your race?" The weighted categorical responses that a participant could choose were: white, black, American Indian or Alaskan native, Asian, native Hawaiian or other pacific islander, other race, multiracial, Hispanic, don't know/not sure, or refused.

\section{COPD and Depression}

For the purposes of this study a participant was considered as having the diagnosis of COPD when the following question was answered as yes: "Have you ever been told you have Chronic Obstructive Pulmonary Disease or COPD, emphysema or chronic bronchitis?" Likewise, a participant was considered as having the diagnosis of depression when the following question was answered as yes: "Have you ever been told that you have a depressive disorder, including depression, major depression, dysthymia, or minor depression?"

\section{Health Related Quality of Life}

In 2016, the BRFSS questionnaire contained 4 questions related to HRQL. Participants were first asked to self-report their health status as excellent, very good, good, fair, or poor. Then, participants were asked to answer the following three questions regarding their HRQL: 1) "Now thinking about your mental health, which includes stress, depression, and problems with emotions, for how many days during the past 30 days was your mental health not good?" 2) "Now thinking about your mental health, which includes stress, depression, and problems with emotions, for how many days during the past 30 days was your mental 
health not good?" 3) "During the past 30 days, for about how many days did poor physical or mental health keep you from doing your usual activities, such as self-care, work, or recreation?" Participants' responses were scored as a continuous variable that ranged from 0 - 30 days.

\section{Smoking Status}

In 2016, the BRFSS questionnaire contained 4 questions related to participants' smoking status. Participants were asked to answer the following questions regarding their smoking status:

1) "Have you smoked at least 100 cigarettes in your entire life?" with answer options of yes or no?

2) "During the past 12 months, have you stopped smoking for one day or longer because you were trying to quit smoking?” The categorical responses that participants could choose were: yes, no, don't know/not sure, or refused.

3) "Do you now smoke cigarettes every day, some days, or not at all?" The categorical responses that participants could choose were: every day, some days, not at all, don't know/not sure, or refused.

4) "How long has it been since you last smoked a cigarette, even one or two puffs?" The categorical responses that participants could choose were: past month, past 3 months, past 6 months, past year, past 5 years, past 10 years, 10 years or more, never smoked regularly, don't know/not sure, or refused.

Depending on participants' answers to question 1 and question 3, the BRFSS calculated and weighted participants smoking status into the following four levels: every day smoker, some day smoker, former smoker, or non-smoker.

\section{Data Analysis}

Data were analyzed using Statistical Package for the Social Sciences 24. Initially, data exploration was completed seeking outliers and missing data. Participants who answered "don't know/not sure" or "refused" to the BRFSS questions were removed from the analyses that included that particular question. Due to the large data set and the small amount of missing data, no manipulation to the missing data occurred. First, a comprehensive descriptive analysis was completed for each variable. This was followed by comparative testing based on variable type. Chi-square tests for independence were used to explore the relationships between categorical study variables of general health related quality of life, depression, and smoking status. Post-hoc tests were used to further characterize. Independent-samples t-tests were used to compare the continuous HRQL variables between participants who were depressed verses those who were not depressed. Mean comparisons of the continuous HRQL variables were conducted using one-way between-groups ANOVA based on the depression and smoking status groups. Post-hoc tests were used as appropriate to further characterize variable relationships.

\section{Results}

The 40,682 individual participants with COPD included 15,271 men (37.5\%) and 
25,406 women $(62.5 \%)$. The majority were 65 years or older $(51.5 \%)$, white (79.8\%), and low income (63.7\% ranging from less than $\$ 10,000$ to less than $\$ 35,000)$. Nearly half of the participants had attended 1 year or more of college (49.1\%) and, nearly all participants reported having health care coverage (94\%). Additional demographic descriptors are displayed in Table 1 and Table 2.

Table 1. Sample characteristics of COPD population, $\mathrm{n}=40,682$.

\begin{tabular}{|c|c|}
\hline Variable & $N,(\%)$ \\
\hline \multicolumn{2}{|l|}{ Gender } \\
\hline Male & $15,271(37.5)$ \\
\hline Female & $25,406(62.5)$ \\
\hline \multicolumn{2}{|l|}{ Age } \\
\hline $18-24$ & $540(1.3)$ \\
\hline $25-34$ & $1265(3.1)$ \\
\hline $35-44$ & $2093(5.1)$ \\
\hline $45-54$ & $5153(12.7)$ \\
\hline $55-64$ & $10,662(26.2)$ \\
\hline 65 or older & $20,969(51.5)$ \\
\hline \multicolumn{2}{|l|}{ Race } \\
\hline White & $32,450(81.2)$ \\
\hline Black & $3093(7.7)$ \\
\hline American Indian/Alaskan Native & $869(2.2)$ \\
\hline Asian & $219(0.5)$ \\
\hline Native Hawaiian/Pacific Islander & $81(0.2)$ \\
\hline Other & $185(0.5)$ \\
\hline Multiracial & $1173(2.9)$ \\
\hline Hispanic & $1871(4.7)$ \\
\hline \multicolumn{2}{|l|}{ Income } \\
\hline$<10,000$ & $3603(10.7)$ \\
\hline$<15,000$ & $4104(12.2)$ \\
\hline$<20,000$ & $4608(13.7)$ \\
\hline$<25,000$ & $4685(13.9)$ \\
\hline$<35,000$ & $4463(13.2)$ \\
\hline$<50,000$ & $4554(13.5)$ \\
\hline$<75,000$ & $3648(10.8)$ \\
\hline 75,000 or more & $4080(12.1)$ \\
\hline \multicolumn{2}{|l|}{ Education Level } \\
\hline Never Attended/Only Kindergarten & $69(0.2)$ \\
\hline Grades 1 - 8 & $1729(4.3)$ \\
\hline Grades $9-11$ & $4361(10.8)$ \\
\hline Grade 12 or GED & $14,491(35.7)$ \\
\hline College 1 - 3 years & $12,083(29.8)$ \\
\hline College 4 or more years & $7818(19.3)$ \\
\hline \multicolumn{2}{|l|}{ Access to Health Care } \\
\hline Yes & $38,221(94.3)$ \\
\hline No & $2324(5.7)$ \\
\hline
\end{tabular}


Table 2. Self-reported sample description.

\begin{tabular}{|c|c|c|c|}
\hline Variables & $N,(\%)$ & & \\
\hline \multicolumn{4}{|l|}{ HRQL General Health Frequencies } \\
\hline Excellent & $1368(3.4)$ & & \\
\hline Very Good & $5512(13.6)$ & & \\
\hline Good & $12,128(29.9)$ & & \\
\hline Fair & $12,594(31.1)$ & & \\
\hline Poor & $8923(22)$ & & \\
\hline \multicolumn{4}{|l|}{ Interval Since Last Smoked } \\
\hline$<1$ month & $411(2.5)$ & & \\
\hline 1 month but less than 3 months & $373(2.2)$ & & \\
\hline 3 months but less than 6 months & $399(2.4)$ & & \\
\hline Within the past year & $637(3.8)$ & & \\
\hline Within the past 5 years & $2677(16.1)$ & & \\
\hline Within the past 10 years & $2129(12.8)$ & & \\
\hline 10 years or more & $9976(59.9)$ & & \\
\hline Never smoked & $61(0.4)$ & & \\
\hline \multicolumn{4}{|l|}{ Smoking Status } \\
\hline Every day & $9737(24.8)$ & & \\
\hline Some days & $3027(7.7)$ & & \\
\hline Former & $16,789(42.7)$ & & \\
\hline Never & $9723(24.8)$ & & \\
\hline HRQL Continuous Descriptive Variables & $\mathbf{N}$ & Mean & SD \\
\hline Physical health not good & 39,233 & 11.7 & 12.5 \\
\hline Mental health not good & 39,655 & 7.1 & 11 \\
\hline Poor physical or mental health & 29,787 & 10.6 & 12.1 \\
\hline
\end{tabular}

To answer research question 1, "For patients with COPD, is there a difference in HRQL for COPD patients who have depression when compared to those who do not have depression?" independent-samples two tailed t-tests are reported. Comparing the number of days during the past 30 days participants physical health was not good, participants with COPD alone $(M=9.4, S D=11.9)$ reported a lower mean number of days when physical health was not good when compared to those who had a dual diagnosis of COPD and depression $(M=$ $15.5, S D=12.5 ; t(28,719.2)=47.3, p<0.01)$. Comparing the number of days participants mental health was not good, participants with the dual diagnosis of COPD and depression had more days when mental health was not good $(M=$ 14.2, $S D=12.4 ; t(20,106.4)=99.0, p<0.01)$ when compared to those with COPD alone $(M=3.0, S D=7.3$, Cohen's $\mathrm{d}=0.6)$. Comparing the number of days participants physical or mental health kept them from performing their 
usual activities, those with a dual diagnosis of COPD and depression $(M=13.8$, $S D=12.3 ; t(26,846.8)=41.0, p=0.0$, two-tailed $)$ reported a higher mean number of days with difficulty when compared to those with COPD alone $(M=8.1$, $S D=11.3$, Cohen's $\mathrm{d}=0.3)$. These findings indicate that hypothesis 1 was supported.

To answer research question 2, "What is the difference in the smoking status in COPD patients who have depression when compared to those who do not have depression?", chi-square tests for independence (with Yates' Continuity Correction) were conducted. The detailed results of each chi-square analysis can be found in Table 3. These results indicate that hypothesis 2 was supported.

To answer the research question 3, "What is the relationship between HRQL and smoking status for a subgroup of patients with COPD and Depression?", cases were selected that met the criteria of the subgroup and then chi-square tests for independence (with Yates' Continuity Correction) were performed. For each smoking status variable, the groups differed significantly on HRQL $(p<0.01)$. Over $80 \%$ of those in the group that smoked over 100 cigarettes rated their health as poor. Over $40 \%$ of the subgroup were still everyday smokers yet just over $43 \%$ of the everyday smokers still rated their health as excellent. Over $65 \%$ of those who quit smoking in the 12 months prior to data collection still reported their health as poor. Table 4 illustrates the details of the chi-square analysis.

Independent-samples t-tests were conducted to compare the continuous HRQL variables of physical health, mental health, and poor health with the dichotomous smoking status variables for patients with a dual diagnosis of COPD and depression. Participants who had stopped smoking in the past 12 months reported significantly higher means for physical health $(p<0.05)$ and poor

Table 3. Chi-square analysis: Number of cigarettes smoked for COPD with and without depression.

\begin{tabular}{cccc}
\hline Variables & No Depression & Depression & $\mathbf{x}^{2}, p$ value \\
\hline Smoking status & & & $957.4, p=0.000^{* *}$ \\
Every day & $5074(20.5)$ & $4599(32)$ & \\
Some days & $1567(6.3)$ & $1438(10)$ & \\
Former smoker & $11,510(46.6)$ & $5191(36.2)$ & \\
Non-smoker & $6543(26.5)$ & $3122(21.8)$ & \\
Days now smoking & & & \\
Every day & $5074(28)$ & $4599(41)$ & \\
Some days & $1567(8.6)$ & $1438(12.8)$ & \\
Not at all & $11,510(63.4)$ & $5191(46.2)$ & \\
Smoked 100 cigarettes & & & \\
Yes & $18,193(73.5)$ & $11,253(78.3)$ & \\
No & $6543(26.5)$ & $3122(21.7)$ & \\
\hline
\end{tabular}

**indicates statistical significance. 
Table 4. Chi-square analysis: categorical HRQL variable and smoking variables.

\begin{tabular}{|c|c|c|c|c|c|c|}
\hline \multirow{2}{*}{ Variables } & \multicolumn{5}{|c|}{ General Health N (\%) } & \multirow{2}{*}{$\begin{array}{c}\mathbf{x}^{2} \\
p \text { value }\end{array}$} \\
\hline & Excellent & Very Good & Good & Fair & Poor & \\
\hline Smoking status & & & & & & $\begin{array}{c}168.4 \\
p=0.000\end{array}$ \\
\hline Every day & $72(27)$ & $287(25)$ & $1108(32.9)$ & $1670(33.6)$ & $1449(31.8)$ & \\
\hline Some days & $14(5.2)$ & $83(7.2)$ & $279(8.3)$ & $520(10.5)$ & $538(11.8)$ & \\
\hline Former smoker & $81(30.3)$ & $424(37)$ & $1206(35.8)$ & $1752(35.3)$ & $1709(37.6)$ & \\
\hline Non-smoker & $100(37.5)$ & $353(30.8)$ & $773(23)$ & $1025(20.6)$ & 855 & \\
\hline Days now smoking & & & & & & $\begin{array}{c}46.9 \\
p=0.000\end{array}$ \\
\hline Every day & $72(43.1)$ & $287(36.1)$ & $1108(42.7)$ & $1670(42.4)$ & $1449(39.2)$ & \\
\hline Some days & $14(8.4)$ & $83(10.5)$ & $279(10.8)$ & $520(13.2)$ & $538(14.6)$ & \\
\hline Not at all & $81(48.5)$ & $424(53.4)$ & $1206(46.5)$ & $1752(44.4)$ & $1709(46.2)$ & \\
\hline Smoked 100 cigarettes & & & & & & $\begin{array}{c}123.8 \\
p=0.000\end{array}$ \\
\hline Yes & $167(62.5)$ & $797(69.3)$ & $2597(77.1)$ & $3950(79.4)$ & $3706(81.3)$ & \\
\hline No & $100(37.5)$ & $353(30.7)$ & $773(22.9)$ & $1025(20.6)$ & 855 & \\
\hline Stopped Smoking & & & & & & $\begin{array}{c}19.6 \\
p=0.001\end{array}$ \\
\hline Yes & $47(54.7)$ & $226(61.4)$ & $822(59.4)$ & $1416(64.8)$ & $1300(65.7)$ & \\
\hline No & $39(45.3)$ & $142(38.6)$ & $563(40.6)$ & $770(35.2)$ & $678(34.3)$ & \\
\hline Last Smoked & & & & & & $\begin{array}{c}55.8 \\
p=0.001\end{array}$ \\
\hline Past month & $4(4.9)$ & $18(4.3)$ & $43(3.6)$ & $65(3.7)$ & $84(5)$ & \\
\hline Past 3 months & $2(2.5)$ & $8(1.9)$ & $28(2.3)$ & $62(3.6)$ & $64(3.8)$ & \\
\hline Past 6 months & $7(8.6)$ & $12(2.8)$ & $33(2.7)$ & $53(3)$ & $71(4.2)$ & \\
\hline Past year & $6(7.4)$ & $18(4.3)$ & $53(4.4)$ & $76(4.4)$ & $102(6)$ & \\
\hline Past 5 years & $14(17.3)$ & $67(15.8)$ & $216(18)$ & $322(18.5)$ & $355(21)$ & \\
\hline Past 10 years & $10(12.3)$ & $58(13.7)$ & $156(13)$ & $236(13.6)$ & $223(13.2)$ & \\
\hline 10 years or more & $38(46.9)$ & $242(57.2)$ & $670(55.7)$ & $921(52.9)$ & $790(46.6)$ & \\
\hline
\end{tabular}

health $(p<0.01)$. The groups did not differ significantly on mental health. When compared for number of cigarettes, the groups who had smoked at least 100 cigarettes had higher mean scores on the physical health $(p<0.01)$, mental health $(p<0.01)$, and poor health variable $(p<0.01)$ indicating that this group had more days when they did not feel well. Table 5 illustrates the details of the independent-samples t-tests.

A one-way between-groups analysis of variance was conducted to explore the relationship between the frequency of day now smoking and the continuous HRQL variables; physical health, mental health, and poor physical or mental 
Table 5. Mean score comparisons for HRQL continuous variables and smoking status 2 group variables.

\begin{tabular}{ccccccc}
\hline \multirow{2}{*}{ Variables } & \multicolumn{9}{c}{ Yes } & \multicolumn{2}{c}{ No } & \multirow{2}{*}{$t$} \\
\cline { 2 - 5 } & Mean & SD & Mean & SD & & \\
\hline Stopped Smoking & & & & & & \\
Physical health & 16.7 & 12.3 & 16.0 & 12.6 & 2 & 0.05 \\
Mental health & 16.7 & 12.1 & 16.1 & 12.4 & 1.7 & 0.09 \\
Poor health & 15.2 & 12.2 & 14.1 & 12.4 & 3.1 & 0.002 \\
Smoked 100 Cigarettes & & & & & & \\
Physical health & 16 & 12.5 & 14 & 12.2 & 7.9 & 0.000 \\
Mental health & 14.7 & 12.4 & 12.6 & 12.1 & 8.4 & 0.000 \\
Poor health & 14.2 & 12.3 & 12.3 & 12.2 & 7 & 0.000 \\
\hline
\end{tabular}

health. Participants were divided into 3 groups according to their frequency of smoking (every day smoker; someday smoker; not at all smoker). There was a statistically significant difference at the $p<0.01$ level in the HRQL variable scores for the three-smoking groups: physical health $F=15.5, p<0.01$; mental health $F=135.3, p<0.01$, poor health $F=22.6, p<0.01$. ANOVA results and Post-hoc comparisons using the Tukey HSD test can be found in Table $6 \&$ Table 7.

A one-way between-groups analysis of variance was conducted to explore the impact of the interval since last smoked on the HRQL continuous variables; physical health, mental health, and poor physical or mental health. Participants were divided into 8 groups according to their interval since last smoked (past month; past 3 months; past 6 months; past year; past 5 years; past 10 years; 10 years or more; never smoked). There was a statistically significant difference at the $p<0.05$ level in the HRQL variable scores for the eight interval groups: physical health $F=6.6, p=0.000$; mental health $F=15.6, p=0.000$, poor health $F=7.6, p=0.000$. Despite reaching statistical significance, the actual difference in mean scores between the groups was small. The effect size, calculated using eta squared was: physical health $=0.009$; mental health $=0.02$; poor health $=$ 0.01 . For the HRQL variables, physical health and mental health, those who last smoked in the past three months had the highest means $(M=19.4 \& 16.9)$. For the variable, poor physical or mental health, those who last smoked in the past 6 months had the highest mean $(M=16.9)$. In all three HRQL variables, those who last smoked 10 years or more had the lowest means. ANOVA results and Post-hoc comparisons using the Tukey HSD test can be found in Table 6 \& Table 7.

A one-way between-groups analysis of variance was conducted to explore the impact of smoker status on the HRQL continuous variables; physical health, mental health, and poor physical or mental health. Participants were divided into 4 groups according to their interval since last smoked (every day smoker; 
Table 6. ANOVA results.

\begin{tabular}{cccccc}
\hline Variable & $d f$ & Sum of Squares & Mean Square & $f$ & $p$ value \\
\hline Frequency of days smoking & 2 & & & & 0.000 \\
Physical health & & 4844.5 & 2422.2 & 15.5 & \\
Mental health & $40,650.5$ & $20,325.3$ & 135.3 & \\
Poor health & 7 & 6830.6 & 3415.3 & 22.6 & \\
Interval since last smoked & & & & & 0.000 \\
Physical health & & 7304.1 & 1043.4 & 6.6 & \\
Mental health & $16,085.4$ & 2297.9 & 15.6 & \\
Poor health & 8093 & 1156.1 & 7.6 & \\
Smoker status & 3 & & & & 0.000 \\
Physical health & & $14,294.6$ & 4764.9 & 30.7 & \\
Mental health & $51,266.9$ & 17,089 & 114.4 & \\
Poor health & $14,114.3$ & 4704.8 & 31.2 & \\
\hline
\end{tabular}

Table 7. Post-Hoc Tukey results.

\begin{tabular}{|c|c|c|c|}
\hline HRQL variables & Frequency of days now smoking & Mean & SD \\
\hline \multirow[t]{3}{*}{ Physical health } & Everyday & 16.1 & 12.5 \\
\hline & Someday & 17.4 & 12.1 \\
\hline & Not at all & 15.3 & 12.6 \\
\hline \multirow[t]{3}{*}{ Mental health } & Everyday & 16.5 & 12.3 \\
\hline & Someday & 16.4 & 12.1 \\
\hline & Not at all & 12.6 & 12.3 \\
\hline \multirow[t]{4}{*}{ Poor health } & Everyday & 14.4 & 12.3 \\
\hline & Someday & 15.9 & 12.1 \\
\hline & Not at all & 13.4 & 12.4 \\
\hline & Interval since last smoked & & \\
\hline \multirow[t]{8}{*}{ Physical health } & Past month & 17.6 & 12.4 \\
\hline & Past 3 months & 19.4 & 12.1 \\
\hline & Past 6 months & 17.6 & 12.3 \\
\hline & Past year & 16.6 & 12.3 \\
\hline & Past 5 years & 15.8 & 12.7 \\
\hline & Past 10 years & 15.3 & 12.8 \\
\hline & 10 years or more & 14.4 & 12.6 \\
\hline & Never smoked & 15.4 & 12.7 \\
\hline \multirow[t]{3}{*}{ Mental health } & Past month & 16.2 & 12.5 \\
\hline & Past 3 months & 16.9 & 12.2 \\
\hline & Past 6 months & 14.2 & 12.2 \\
\hline
\end{tabular}




\section{Continued}

\begin{tabular}{|c|c|c|c|}
\hline & Past year & 15.8 & 12.6 \\
\hline & Past 5 years & 14.1 & 12.6 \\
\hline & Past 10 years & 11.9 & 12.0 \\
\hline & 10 years or more & 11.2 & 12.0 \\
\hline & Never smoked & 14.6 & 12.2 \\
\hline \multirow[t]{9}{*}{ Poor health } & Past month & 14.2 & 12.2 \\
\hline & Past 3 months & 16.4 & 12.3 \\
\hline & Past 6 months & 16.9 & 12.4 \\
\hline & Past year & 15.7 & 12.6 \\
\hline & Past 5 years & 14.4 & 12.5 \\
\hline & Past 10 years & 12.9 & 12.3 \\
\hline & 10 years or more & 12.4 & 12.2 \\
\hline & Never smoked & 15.3 & 11.3 \\
\hline & Smoker Status & & \\
\hline \multirow[t]{4}{*}{ Physical health } & Everyday & 16.1 & 12.5 \\
\hline & Someday & 17.4 & 12.1 \\
\hline & Former & 15.3 & 12.6 \\
\hline & Non-Smoker & 13.9 & 12.2 \\
\hline \multirow[t]{4}{*}{ Mental health } & Everyday & 16.5 & 12.3 \\
\hline & Someday & 16.4 & 12.1 \\
\hline & Former & 12.6 & 12.3 \\
\hline & Non-Smoker & 12.3 & 12.1 \\
\hline \multirow[t]{4}{*}{ Poor health } & Everyday & 14.4 & 12.3 \\
\hline & Someday & 15.9 & 12.1 \\
\hline & Former & 13.4 & 12.4 \\
\hline & Non-Smoker & 12.3 & 12.2 \\
\hline
\end{tabular}

someday smoker; former smoker; non-smoker). There was a statistically significant difference at the $p<0.05$ level in the HRQL variable scores for the four groups: physical health $F=30.7, p=0.000$; mental health $F=114.4, p=0.000$, poor health $F=31.2, p=0.000$. Despite reaching statistical significance, the actual difference in mean scores between the groups was quite small. The effect size, calculated using eta squared was: physical health $=0.007$; mental health $=$ 0.02 ; poor health $=0.007$. ANOVA results and Post-hoc comparisons using the Tukey HSD test can be found in Table 6 \& Table 7. These results indicate that hypothesis 3 was supported.

\section{Discussion}

The prevalence of COPD in the population was $8.3 \%$, which is higher than the 
reported national prevalence rate of 6.4\% [1]. Previously, in 2013, American Indians, Alaskan natives, and multiracial non-Hispanics were disproportionally diagnosed with COPD; however, in this weighted COPD sample, $81.2 \%$ of participants identified as being white. There were also $25 \%$ more women diagnosed with COPD than men in this population. These findings are consistent with the literature over the last decade which finds women self-reporting their COPD diagnoses more often than men [2]. More than half of the sample was 65 years or older, $94 \%$ of participants had healthcare coverage, and $79.8 \%$ fell within the low-income level.

Prevalence of COPD correlates with those individuals who are 65 years and older [1]. With an older population and $94 \%$ of participants having healthcare coverage, one can assume that Medicare was the primary source of insurance. Prevalence of COPD is also known to have an inverse relationship with income [2]. Additionally, it is known that big tobacco targets lower income individuals, and the proportion of smokers developing COPD is now at 50\% [2]. Lower educational obtainment is correlated with COPD and smoking which is consistent with the findings of this study as nearly half of the participants attended one year of college or less [1].

The 36\% prevalence rate of depression within this sample correlates with the overall prevalence rate of depression among COPD patients (40\%) [5]. The majority of the participants reported their HRQL as good or less (83\%), with only a small percent of the sample describing their quality of life as very good or excellent $(17 \%)$. In addition, participants reported a mean of seven or more days with poor physical health, poor mental health, and poor health per month. These results support the notion that COPD has a profound effect on patients HRQL; however, participants with COPD and depression reported poorer HRQL. This subgroup reported a higher mean number of days their physical health, mental health and poor health were not good compared to those participants with COPD. These findings supported hypothesis 1.

The majority of participants with COPD (73.5\%) and the majority of participants with COPD and depression (78.3\%) had all smoked at least 100 cigarettes in their life. Considering that smoking is the leading risk factor for COPD and accounts for $90 \%$ of COPD diagnoses, this finding was consistent with the literature [6]. However, for patients with COPD, the number of cigarettes smoked is greater for those with depression than those without depression. This finding is consistent with the literature, as the prevalence of smoking increases in patients with mental disorders [9]. A greater percentage of participants with depression (32\%) reported their smoking status as an "every day smoker", in comparison to participants with COPD alone who reported their smoking status as a "former smoker". When reporting their days now smoking, a larger percentage of those with depression reported smoking every day and those with COPD alone reported not smoking at all. These findings supported hypothesis 2 .

These findings are consistent with the literature that nicotine dependence plays an important role in the relationship between COPD and depression [9]. It 
also indicates that depression may have a causative influence on the choice to continue smoking. Future research should be aimed at increasing screening and treatment for depression in patients with COPD who continue to smoke. Such screening and intervention may lead to improved outcomes. From a clinical perspective, if nicotine dependence is a confounding variable between COPD and depression, there is a highlighted need for health behavior counseling and treatment for nicotine dependence in this population [9].

Health related quality of life seems to be impacted by smoking status for participants with COPD and depression. More nonsmokers rated their health as excellent, compared to the majority of everyday smokers who rated their health as good, fair, or poor. In addition, a larger percentage of participants who reported their general health as excellent were not currently smoking at all compared to those who were currently smoking every day. These findings supported hypothesis 3. However, there was an unexpected finding in this analysis. Individuals that had stopped smoking, but not achieved smoking cessation for five years, reported a higher number of days with poor physical and mental health and a higher number of days that their overall health impacted their activities of daily living. Non-smokers do rate their physical, mental, and overall health higher compared to those participants that smoke every day. Those who have achieved smoking cessation for five years or more report better physical, mental, and poor health. This is consistent with the finding that quality of life improves when a patient achieves successful smoking cessation [10].

However, those individuals who last smoked within the past three months reported poor physical health with a mean of 19.4 days. The increase in this mean may be due to patients experiencing symptoms of acute nicotine withdrawal [11]. Non and former smokers have better HRQL compared to every day smokers; however, someday smokers had the highest means for physical and poor health. We hypothesize that smoking may be a coping mechanism that improves participants perceived quality of life especially during acute nicotine withdrawal or because of acute nicotine withdrawal. If this is to be true, this finding highlights the importance of evaluating coping mechanisms and smoking cessation interventions by healthcare professionals. Hence, there is a need to investigate the link between depression and unsuccessful smoking cessations attempts for people with COPD.

\section{Limitations}

While the BRFSS dataset is a large nationally representative sample, this study does have limitations. Performing a secondary analysis on the BRFSS dataset, limited the researcher to the sampling procedures and the data provided. Due to the cross-sectional design, no causal relationships can be determined between COPD, depression, and HRQL. The BRFSS is a telephone survey, which may exclude potential participants that do not have telephones. Additionally, all of the data collected was self-reported by the participants. Smoking status and the di- 
agnoses of COPD and/or depression were not validated otherwise. Likewise, COPD and depression are often underdiagnosed which could lead to a misrepresented sample.

\section{Future Implications and Conclusion}

The causal relationship between COPD and smoking is well understood; however, the causal relationship between COPD, depression, and smoking cessation is not well understood. This study finds depression, as a comorbidity, does relate to differences in smoking status and physical, mental, and overall HRQL. The results of this study provide further evidence that it is critical to assess for depression in persons with COPD. These results are foundational to a developing program of research focused on the potentially cyclical nature of the relationships among COPD, depression, smoking status, and HRQL is planned. Identifying and understanding these relationships will be key to the development of interventions targeting smoking cessation in this population.

\section{Funding}

Jonas Scholar 2018-2020.

\section{Conflicts of Interest}

The authors declare no conflicts of interest regarding the publication of this paper.

\section{References}

[1] Centers for Disease Control and Prevention (2016) Chronic Obstructive Pulmonary Disease. https://www.cdc.gov/copd/data.html

[2] Rosenberg, S.R., Kalhan, R. and Mannino, D.M. (2015) Epidemiology of Chronic Obstructive Pulmonary Disease: Prevalence, Morbidity, Mortality, and Risk Factors. Seminars in Respiratory and Critical Care Medicine, 36, 457-469. https://doi.org/10.1055/s-0035-1555607

[3] Christenhusz, L.C.A., Prenger, R., Pieterse, M.E., Seydel, E.R. and van der Palen, J. (2012) Cost-Effectiveness of an Intensive Smoking Cessation Intervention for COPD Outpatients. Nicotine \& Tobacco Research, 14, 657-663. https://doi.org/10.1093/ntr/ntr263

[4] Hilberink, S.R., Jacobs, J.E., Bottema, B.J.A.M., de Vries, H. and Grol, R.P.T.M. (2005) Smoking Cessation in Patients with COPD in Daily General Practice (SMOCC): Six Months' Results. Preventive Medicine: An International Journal Devoted to Practice and Theory, 41, 822-827. https://doi.org/10.1016/j.ypmed.2005.08.003

[5] Yohannes, A.M., Hann, M. and Sibbald, B. (2011) The Management of Depressive Symptoms in Patients with COPD: A Postal Survey of General Practitioners. Primary Health Care Research and Development, 12, 237-244. https://doi.org/10.1017/S1463423611000041

[6] Bratek, A., Zawada, K., Beil-Gawełczyk, J., Beil, S., Sozańska, E., Krysta, K., et al. (2015) Depressiveness, Symptoms of Anxiety and Cognitive Dysfunctions in Patients with Asthma and Chronic Obstructive Pulmonary Disease (COPD): Possible 
Associations with Inflammation Markers: A Pilot Study. Journal of Neural Transmission, 122, 83-91. https://doi.org/10.1007/s00702-014-1171-9

[7] Rapsey, C.M., Lim, C.C.W., Al-Hamzawi, A., Alonso, J., Bruffaerts, R., Caldas-de-Almeida, J.M., et al. (2015) Associations between DSM-IV Mental Disorders and Subsequent COPD Diagnosis. Journal of Psychosomatic Research, 79, 333-339. https://doi.org/10.1016/j.jpsychores.2015.08.005

[8] CDC (2017) Behavioral Risk Factor Surveillance System Overview: BRFSS 2016. https://www.cdc.gov/brfss/annual_data/2016/pdf/overview_2016.pdf

[9] Goodwin, R.D., Lavoie, K.L., Lemeshow, A.R., Jenkins, E., Brown, E.S. and Fedoronko, D.A. (2012) Depression, Anxiety, and COPD: The Unexamined Role of Nicotine Dependence. Nicotine \& Tobacco Research, 14, 176-183.

https://doi.org/10.1093/ntr/ntr165

[10] Christenhusz, L., Pieterse, M., Seydel, E. and van der Palen, J. (2007) Prospective Determinants of Smoking Cessation in COPD Patients within a High Intensity or a Brief Counseling Intervention. Patient Education and Counseling, 66, 162-166. https://doi.org/10.1016/j.pec.2006.11.006

[11] Paul, M., Cinciripini, P.J.D.R., Karam-Hage, M., Minnix, J.A., Lam, C., Francesco Versace, P.V.L.B., Engelmann, J.M. and Wetter, D.W. (2013) Effects of Varenicline and Bupropion Sustained-Release Use Plus Intensive Smoking Cessation Counseling on Prolonged Abstinence from Smoking and on Depression, Negative Affect, and Other Symptoms of Nicotine Withdrawal. American Medical Association, Chicago, 522-533. 